\title{
Mechanisms of dwell fatigue crack growth in an advanced nickel disc alloy RR1000
}

\author{
S.Y. Yu ${ }^{1, a}$, H.Y. Li ${ }^{1}$, M.C. Hardy ${ }^{2}$, S.A. McDonald ${ }^{3}$, and P. Bowen ${ }^{1}$ \\ ${ }^{1}$ School of Metallurgy and Materials, College of Engineering and Physical Science, University of Birmingham, \\ Edgbaston, Birmingham B15 2TT, UK \\ 2 Rolls-Royce plc, PO Box 31, Derby DE24 8BJ, UK \\ ${ }^{3}$ Manchester X-ray Imaging Facility, School of Materials, University of Manchester, Manchester M13 9PL, UK
}

\begin{abstract}
RR1000 is one of an advanced class of nickel-based superalloys developed for disc applications. Under one hour dwell fatigue loading, complex crack growth behaviour has been observed especially in a coarse grained version of this alloy. At a temperature of $700{ }^{\circ} \mathrm{C}$ in air an increase of nearly two orders of magnitude in crack growth rates compared to baseline fatigue crack growth rates may be seen. However for certain microstructural conditions, cracks can also demonstrate retardation following initial acceleration. When using a direct current potential difference (d.c.p.d) technique for monitoring crack growth, a damage zone of a few hundred microns is often measured ahead of a fast growing crack. Advanced characterisation techniques including SEM, ECCI and X-ray tomography have been adopted in the current study to understand the observed damage zone and retardation phenomenon. It is found that damage zones measured by d.c.p.d reflect brittle and non-uniform advance of the crack resulting from continuous dynamic or quasi-dynamic fracture of an oxide intrusion ahead of the crack tip during the dwell period. In contrast, cracking of the oxide intrusion is less frequent or even prevented during dwell periods associated with a retarded and slow growing crack. Crack tip stress relaxation plays an important role in dictating whether or not dynamic cracking of the oxide intrusion can be avoided.
\end{abstract}

\section{Introduction}

The continuous drive for improved engine performance in the civil aviation industry has resulted in the development of a new generation of powder processed $\mathrm{Ni}$ based superalloys for high pressure (HP) disc rotor applications. Compared with existing alloys, the new generation of alloys show improved resistance to high temperature plasticity and creep. Increased time dependent dwell crack growth can occur in oxidizing environments at temperatures in excess of $500^{\circ} \mathrm{C}$ as a result of grain boundary oxidation and subsequent embrittlement during the prolonged dwell period at the peak load. This leads to a change in crack growth mechanism from transgranular to intergranular and an increase in crack growth rates [1-5].

The change in behaviour during dwell fatigue crack growth raises a concern for new generation alloys, due to its potential to significantly reduce the damage tolerance life of engine components. Whilst considerable research has been carried out to understand crack growth under dwell fatigue loading, a comprehensive understanding of the micro-mechanisms for environmentally assisted intergranular crack growth is not available. Dynamic Embrittlement [6-8] and Stress Assisted Grain Boundary

\footnotetext{
${ }^{a}$ Corresponding author: SXY182@bham.ac.uk
}

Oxidation (SAGBO) [9] are two main concepts currently proposed.

It has been reported that a damage zone exists ahead of crack tip which is associated with the increase in crack growth rates following dwell fatigue cycles or sustained load $[1,10]$. The damage zone is considered to be a region of material ahead of the crack tip that is weakened by long range oxygen diffusion (a few hundred microns) and oxidation. Such a damage zone is measurable using a direct current potential difference (d.c.p.d.) technique. Increased crack growth rates were measured within the damage zone when fast fatigue cycling was applied immediately after dwell fatigue or sustained loading. In addition intergranular fracture surface morphology was observed [5,9]. However, only very limited oxide intrusions ahead of the dwell fatigue crack tip $(<10$ microns $)$ are observed on 2D metallographic sections $[2,11]$, which is contradictory to the long range diffusion argument. Although long range oxygen diffusion was suggested [5] the measurement technique adopted could not capture the uneven advance of intergranular cracks. A better understanding of the damage zone phenomenon and its implication to dwell fatigue crack growth behaviour is needed.

In addition to the fast growing condition and the damage zone phenomenon, a retardation in dwell fatigue crack growth rates has also been observed under one 
hour dwell fatigue loading [1]. When the crack growth driving force is low, the dwell fatigue crack growth rates increase at the beginning and then gradually decrease to the normal fatigue baseline rate. This retardation phenomenon is associated with changes in the $\gamma$ ' particle size and other factors such as the magnitude of the initial mechanical driving force [1]. Despite large variations in crack growth behaviour, the microstructural variation can be subtle.

Based on the crack tip oxides observed and the two contrasting modes of behaviour, a more comprehensive micro-mechanism has been proposed to explain both acceleration and retardation in dwell fatigue crack growth [1], in which crack advance is believed to be associated with two different manners of rupture behaviour of the oxide intrusion at the crack tip.

To improve the understanding of the micro-mechanism, the current study has included a number of novel techniques to produce a more detailed quasi-in-situ characterisation of crack morphologies, crack tip damage and deformation for the two contrasting types of crack growth behaviour. The observations support the new theory very well.

\section{Experimental}

\subsection{Materials}

The material used in this research is RR1000, a new generation nickel-based superalloy, developed by RollsRoyce plc. for disc rotors in the HP compressor and turbine of aircraft engines. The nominal chemical composition of this material in wt. $\%$ is $15 \mathrm{Cr}, 18.5 \mathrm{Co}$, $5 \mathrm{Mo}, 3 \mathrm{Al}, 3.6 \mathrm{Ti}, 2 \mathrm{Ta}, 0.5 \mathrm{Hf}, 0.06 \mathrm{Zr}, 0.027 \mathrm{C}, 0.015 \mathrm{~B}$ and a balance of $\mathrm{Ni}$. Corner crack fatigue test pieces (with a square $7 \times 7 \mathrm{~mm}^{2}$ cross-section) with a coarse grain microstructure $(\sim 50 \mu \mathrm{m})$ and a bi-modal population of $\gamma$ ' strengthening precipitates were used.

\subsection{Interrupted mechanical tests}

Interrupted fatigue crack growth tests were performed to establish two different dwell fatigue crack states: (1) rapid growing, and (2) slow and retarding. The tests were performed on a screw-driven mechanical testing machine at a temperature of $700{ }^{\circ} \mathrm{C}$ in air. A d.c.p.d technique was applied to monitor the crack length during the tests. Trapezoidal loading waveforms with different dwell times at peak load were applied. The ramping up, ramping down and dwell at minimum load were all set to be 1 second. 1 hour dwell (noted as 1-3600-1-1) was applied after fatigue precracking using 1 second dwell (noted as 1-1-1-1). 11-1-1 waveform is regarded as the baseline fatigue as it results in fully transgranular failure at $700^{\circ} \mathrm{C}$ for the material studied. The load amplitude and stress ratio were kept constant $(\mathrm{R}=0.1)$ during fatigue precracking and dwell cycling. The two crack states were achieved either by using different microstructures (size and distribution of $\gamma$ ') resulting from different cooling rates from the supersolvus heat treatment, or starting the dwell fatigue loading at different $\Delta \mathrm{K}$ values, i.e. $\sim 17 \mathrm{MPa} \sqrt{ } \mathrm{m}$ for a retarding crack and $30 \mathrm{MPa} \sqrt{ } \mathrm{m}$ for a rapidly growing crack. A detailed explanation of the influence of microstructure and the magnitude of initial $\Delta \mathrm{K}$ is provided in Ref [1]. The tests were stopped after a number of 1 hour dwell fatigue cycles without breaking open the test pieces to preserve the crack tip morphology for quasi-in-situ characterisation.

\subsection{Crack characterisation}

After the tests were interrupted, the test pieces were kept intact and removed from testing machine. One set of samples were cut along the diagonal cross-section perpendicular to the crack plane to reveal the profile of the crack. Subsequently, these cross-sections were ground and polished to gain a good surface finish for further characterisation. Characterisation of the 2D crack morphology and crack tip oxides were conducted on a Scanning Electron Microscope (SEM). Electron Channelling Contrast Imaging (ECCI) was applied to study deformation around the crack tip by using the backscatter detector in SEM.

The crack morphology, especially how the crack propagates in 3 dimensions, was found to be critical for understanding the damage zone phenomenon. Therefore $\mathrm{X}$-ray computed tomography (XCT) was carried out to generate $3 \mathrm{D}$ crack morphologies for the two contrasting conditions. The XCT samples with a cross section of $1.5 \times$ $1.5 \mathrm{~mm}^{2}$ containing the cracked region of interest were cut from interrupted test pieces and were scanned using an Xradia microXCT at The University of Manchester. 1501 projections were taken with $150 \mathrm{kV}$ X-rays. The results were subsequently reconstructed and visualized using Avizo.

\section{Results}

\subsection{Crack growth behaviour}

Crack growth rates in the form of crack length increment per cycle $(\mathrm{da} / \mathrm{dN})$ versus alternating stress intensity factor $(\Delta \mathrm{K})$ of the two XCT samples are plotted in Fig. 1. At $700^{\circ} \mathrm{C}$ under 1 hour dwell fatigue loading, crack growth rates (in $\mathrm{da} / \mathrm{dN}$ ) generally increased by more than an order of magnitude over the fatigue baseline crack growth rates for both samples. The one transferred to 1 hour dwell cycling at $\sim 17 \mathrm{MPa} \sqrt{ } \mathrm{m}$ demonstrated a decrease in crack growth rates. The other sample which was transferred to 1 hour dwell cycling at $\sim 28 \mathrm{MPa} \sqrt{ } \mathrm{m}$ kept growing at high rates and formed a damage zone. At the point of interruption, the crack growth rate was about 10 times and 100 times faster than that of baseline crack growth rate at comparative $\Delta \mathrm{K}$ respectively for the two cracking situations. The end points of the two curves represent the conditions at which the tests were interrupted for XCT.

The d.c.p.d curves for the two conditions provide some clues to the crack growth behaviour within a dwell fatigue cycle. It was noticed that the potential drop (p.d.) measurements for the two crack growth conditions were also different at the point of interruption, as illustrated in Fig. 2. For the fast growing condition, the p.d. increased during the dwell period at the peak load and there was a big increase during the loading cycle. For the retarded crack, however, the p.d only increased marginally during the unloading and loading segments and it remained nearly 


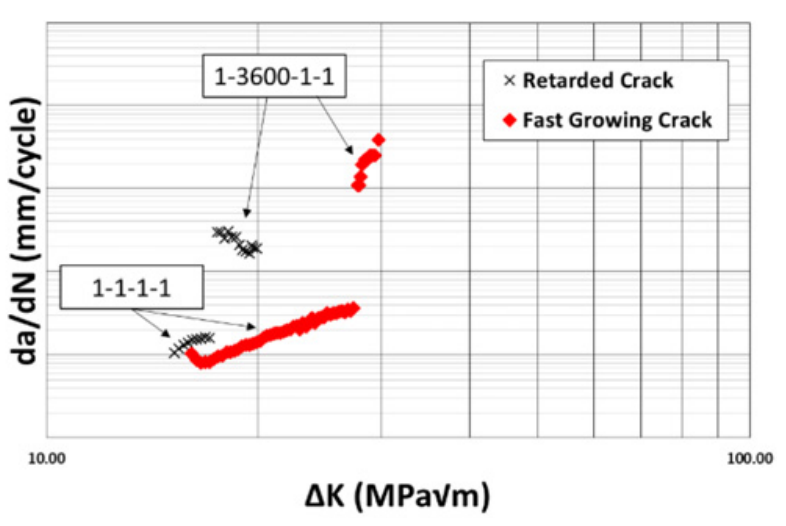

Figure 1. Fatigue 1-1-1-1 baseline crack growth rate and 1 hour dwell fatigue crack growth rate versus $\Delta \mathrm{K}$ curves for the fast growing and retarded conditions, tested at $700{ }^{\circ} \mathrm{C}$.

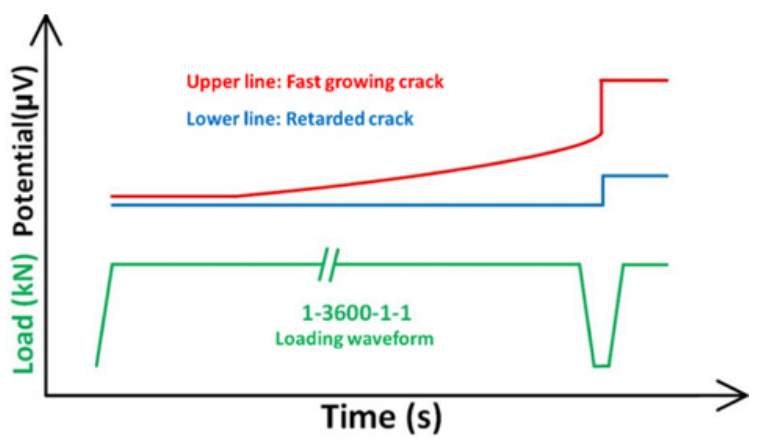

Figure 2. Schematic drawing of crack length change in the form of potential change during 1-3600-1-1 cycle for the two types of crack growth. The green curve represents the loading waveform.

constant during the dwell period. These different curves suggest that the crack growth mechanisms for the two conditions might be different.

\subsection{Crack tip damage}

Damage at the crack tip for the two conditions was characterised after the tests were interrupted. Only a limited oxide intrusion was observed ahead of the crack tip for both conditions regardless of the crack growth rate (Fig. 3). This finding is consistent with other researches' observations using more accurate characterisation techniques, like Atom Probe Tomography (APT) and Transmission Electron Microscope (TEM) $[2,11]$, which also showed no further damage, such as oxide, oxygen or micro cracks, far ahead of the crack tips. These results prove that the depth of oxides/oxygen damage is very limited ahead of open crack front, and obviously much smaller than the damage zone measured by d.c.p.d. This, however, is contrary to the hypothesis that the "damage zone" is a large region of material in which the grain boundaries ahead of crack tip are weakened. The quasi-in-situ direct cross-section observations in this research and the APT and TEM work are much closer to the reality compared with post-mortem XPS measurements on fracture surfaces and fracture surface examination $[5,12]$. This contradiction suggests
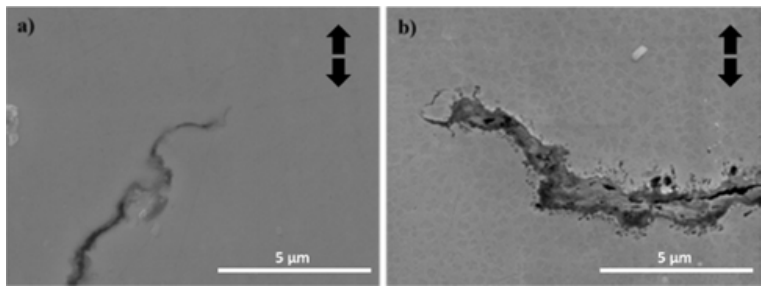

Figure 3. Secondary electron images of crack tip oxides for a) fast growing crack and b) retarded crack in CG microstructures. The arrows indicate the loading direction.

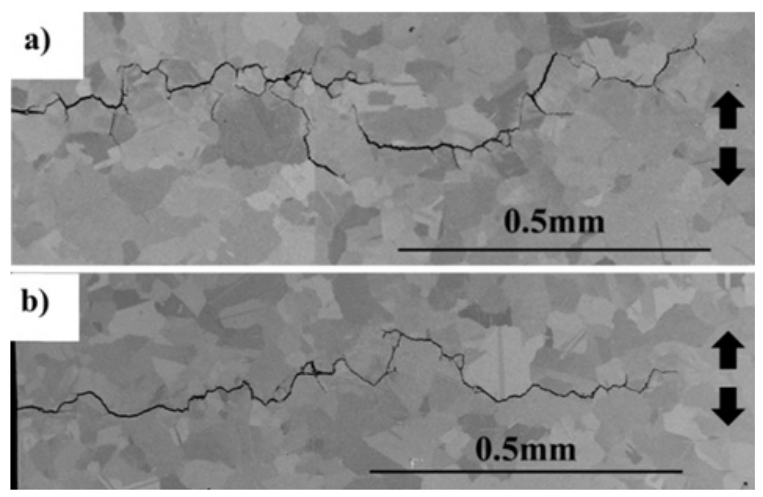

Figure 4. Backscattered electron images showing 2D crosssection view of a) intermittent fast growing crack, and b) continuous retarded crack. The arrows indicate the loading direction.

that damage zone phenomenon may not be the result of long-range oxygen diffusion and oxidation [9].

\subsection{Crack morphologies}

2D crack profiles of the two different crack growth conditions are shown in Fig. 4. The fast growing crack shows segments of cracks although it is believed to grow in a dynamic or near-dynamic manner during dwell [1]. In contrast, the retarded crack is continuous. 3D XCT results show (Fig. 5) that such characteristics for the two crack growth conditions are the case throughout the crack plane.

The "segmental" crack morphology for the fast growing crack is the result of many gaps along the crack which make the crack look discontinuous on a 2D crosssection. These "gaps" are actually un-fractured ligaments which exist behind the furthest extent of the crack. The crack front is still continuous as a whole and there are no cracks detached from the fully separated main crack. The advance of the crack seems to follow a sequence of uneven multiple penetrations like branches along grain boundaries followed by linkage of ligaments. By growing in this fashion oxygen can be transported to the furthest extent of the crack, and the length of the crack front is enlarged significantly compared to the smoother crack front found in the retarded crack. Very few ligaments were found for the retarded crack which is consistent with the $2 \mathrm{D}$ crosssection view.

Disagreement between the real intergranular crack length measured on the $3 \mathrm{D}$ model $(510 \mu \mathrm{m})$, which represents the furthest extent of crack and the crack length indicated by d.c.p.d $(233 \mu \mathrm{m})$ was found for the fast growing crack, as noted in Fig. 5a. The difference 

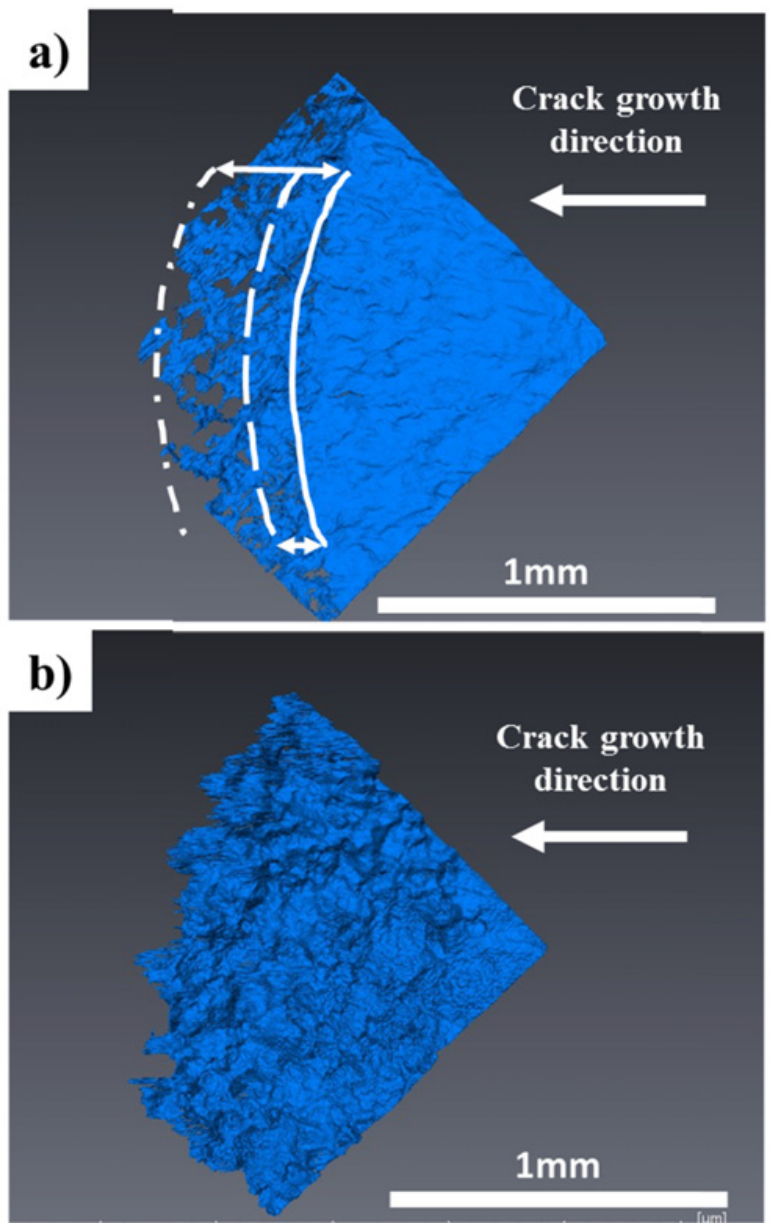

Figure 5. 3D crack morphologies of $1.5 \times 1.5 \mathrm{~mm}^{2}$ crack region for a) fast growing crack and b) retarded crack. The solid curve in a) is the interface between 1-1-1-1 baseline transgranular and 1-3600-1-1 intergranular crack; the dashed curve represents the crack front position indicated by d.c.p.d; the dash dot curve represents the real crack front position.

measured approximately corresponds to the region containing uncracked ligaments ahead of the crack front of complete separation (dashed line in Fig. 5a), and is similar to the size of the damage zone measured at this particular $\Delta \mathrm{K}$ value.

\subsection{Crack tip deformation}

ECCI is a technique very sensitive to crystal orientation due to the channelling effect, which therefore can capture subtle difference in orientation. In an ECC image, the brightness variation (contrast) within each individual grain represents the variation in orientation. As the change in orientation within a single grain could be the result of deformation, this method has been established to study deformation in materials [13]. Fig. 6 shows channelling contrast images around the crack tip for the two featured conditions. These two images were taken without tilting. Note that the samples were also examined with different tilting angles in the SEM, and it was verified that the contrast in these two images can represent the deformation trace for the two conditions.
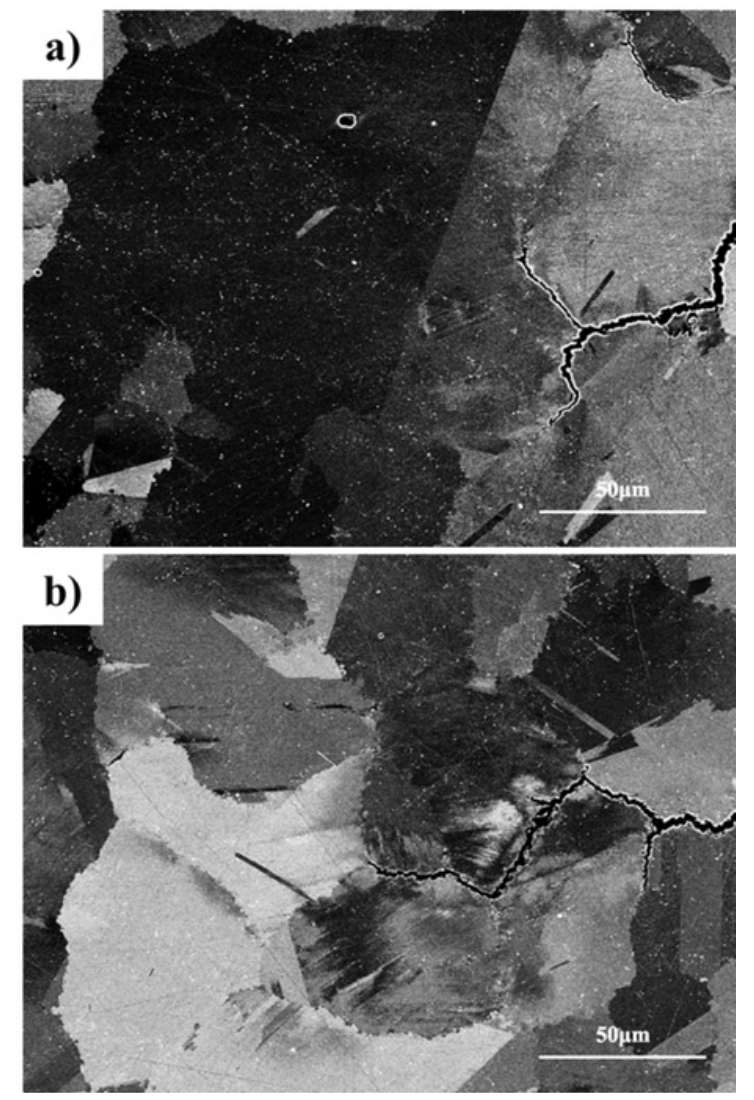

Figure 6. Electron channelling contrast images showing the deformation pattern around the crack tip for a) fast growing crack and b) retarded crack. The latter shows significantly more deformation.

For the fast growing condition in Fig. 6a, there is only a very small variation in brightness within individual grains at the crack tip which indicates that there is very little change in orientation within individual grains. Therefore, the matrix suffers less deformation for this fast growing situation. This observation supports the idea that in this condition such environmentally assisted intergranular fracture is brittle because the cracking is the result of crack tip oxides rupture rather than the deformation of metal matrix.

In contrast, significant variation in brightness within grains around the crack tip is observed on the retarded crack sample (Fig. 6b). Slip bands are clearly seen. Such significant contrast observed should be associated with much more deformation in these grains around the crack tip, which could lead to a larger crack tip opening displacement and therefore crack tip blunting.

\section{Discussion}

\subsection{Damage zone phenomenon}

The initial idea of the damage zone phenomenon was that the dwell fatigue crack was growing along grain boundaries that had been weakened by oxygen and/or oxides induced by previous dwell fatigue cycles $[5,9,12]$. This long range oxygen/oxides damage therefore increased 


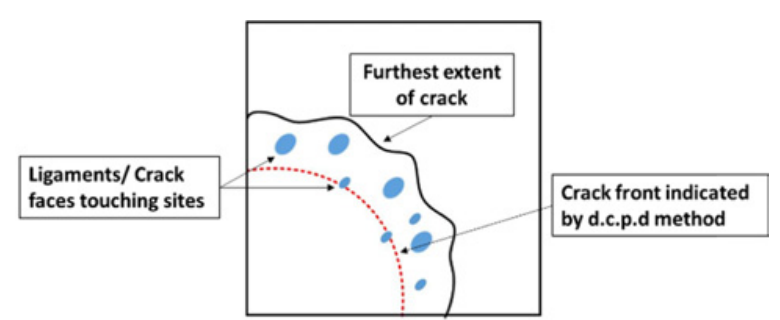

Figure 7. Schematic drawing of how ligaments and crack faces touching affect fast intergranular crack length measurement. The dotted curve represents the crack front position indicated by d.c.p.d which is shorter than the real crack front due to the existence of ligaments behind the real crack front.

the baseline fatigue crack growth rate and changed the baseline fatigue crack growth mechanism from transgranular to intergranular. However, the long range diffusion theory has not been verified because no evidence of oxygen segregation far ahead of the crack tip has been observed using ATP [2] or TEM [11]. Instead, only very limited oxide intrusions were found ahead of the crack tip in these deliberately designed experiments, which suggests that long range oxygen diffusion and consequent grain boundary embrittlement does not exist.

From the current $2 \mathrm{D}$ and $3 \mathrm{D}$ characterisation, it is suggested that the "damage zone" measured by d.c.p.d. represents a zone of special characteristics. As shown in Fig. 5a, it marks a zone between the furthest extent of the crack and an obvious crack front which is indicated by d.c.p.d method, as shown on Fig. 5a. As the d.c.p.d technique is based on the electrical resistance of the whole test piece measured across opposite sides of the crack it usually works well with regular transgranular cracks. However, for dwell fatigue, the lack of sensitivity in recognising all the cracked area may arise from two factors: (1) uncracked ligaments which provide a short cut for the current, and (2) local contact of the crack faces which can occur during holding at peak load due to the brittle nature and uneven advance of intergranular cracks associated with environmental embrittlement. This is demonstrated in Fig. 7.

If the area corresponding to the very furthest extent of the fast growing crack (solid curve in Fig. 7) is A1, and the sum of the area of ligaments and touching crack faces under load is A2 (blue areas in Fig. 7), then only the area A1-A2 contributes to the p.d. reading (dotted curve in Fig. 7), which is therefore apparently smaller than the furthest extent of the crack. This means the crack length indicated by d.c.p.d is shorter than the furthest extent of the crack. Hence when a damage zone is measured it is likely that the furthest extent of the crack is underestimated.

Since the real crack depth (or the furthest extent) of a fast growing intergranular crack is underestimated by d.c.p.d during 1 hour dwell fatigue cycling because of ligaments and touching crack faces, there will appear to be a sharp increase in crack length when the ligaments are broken and the touching crack faces are separated. During each dwell fatigue cycle, the p.d. value increases not only during dwell at peak load but also there is a sudden increase on the unloading and loading segments at the end and beginning of two adjacent dwell fatigue a)

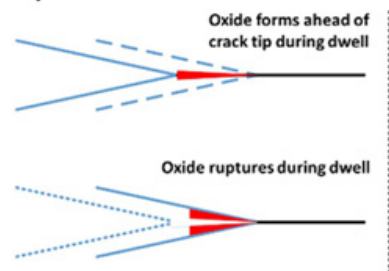

b)

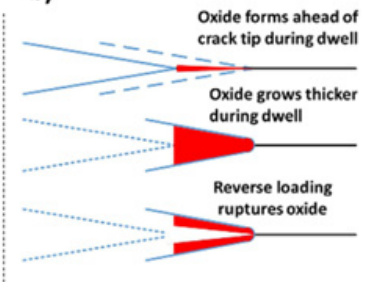

Figure 8. Schematic drawing show the mechanisms for a) continuous dynamic crack growth during dwell and b) cycle-bycycle-only retarded crack growth.

cycles. One fluctuation of load between peak and minimum load helped to partially reveal the underestimated crack length, perhaps by breaking some ligaments and enlarging the crack opening displacement. The full extent of the underestimation of the crack length could be revealed by changing the loading waveform from dwell fatigue to baseline fatigue when all the effects of vulnerable ligaments and touching crack faces are eliminated and the environmental effect is totally replaced by the cyclic stress fatigue mechanism. This is why the fatigue crack growth rates following dwell fatigue cycles are significantly higher than normal, as the fast cycle fatigue was breaking weak ligaments and enlarging crack opening displacement to disclose the whole crack length corresponding to the crack front.

The damage zone phenomenon is now clearer as there is no such zone in which grain boundaries were only weakened by oxygen/or oxides ahead of the crack tip. It is in fact a zone that contains partially intergranularly cracked area. During a holding period at the peak load, it is this zone that moves forward instead of a simple uniform crack front. The appearance of fast fatigue crack growth rates following dwell fatigue cycles, however, represents the elimination of the intergranular cracking process zone by breaking the un-fractured ligaments and expanding the crack opening displacement.

As suggested in Ref. [1], the fast growing crack advances in a brittle and dynamic manner during holding at the peak load. Oxygen diffuses into grain boundaries ahead of crack tip for a very short distance and forms oxides. Because of the high tensile stress at the sharp crack tip in this creep resistant test piece with finer $\gamma$ ', the thin oxide layer ruptures upon forming a very thin intrusion (Fig. 8a). As this cracking is intergranular, the grain boundary type and local normal stress applied on each grain boundary are critical to the crack growth. The type of grain boundary mainly influences the oxygen diffusion and it has been confirmed that high angle grain boundaries are more vulnerable than special boundaries for such intergranular cracking [14]. The local normal stress depends highly on the angle of the grain boundary to the loading direction and the orientation of grains. Thus, the local intergranular crack growth rate could vary dramatically along the crack front and form an irregular crack front relative to a uniform fast cycle crack front. The difference in the local crack front (on the scale of the grain size) will accumulate as time increases. If the time is long enough, 1hour for example, the irregularity of the crack front will be so significant 
that ligaments will form and be left behind the furthest crack front. Hence the damage zone forms. Note that such irregular cracking is not the same as micro-cracks ahead of crack tip which are not connected to the main crack as here the crack is all interconnected as a whole because the intergranular crack is only possible when oxygen can be delivered to the crack tip by open crack path.

\subsection{Retardation phenomenon}

The crack growth mechanism in the case of the retarded crack is believed to be different from the fast growing case [1]. This could be deduced to be due to the adopted loading condition (low initial $\Delta \mathrm{K}$ ) and/or microstructure (coarser secondary and tertiary $\gamma^{\prime}$ ) which could lead to accumulation of local creep deformation at the crack tip. Hence the crack tip may be blunted which would lead to a redistribution and lowering of the stress at the crack tip. Such a change in the stress field at the crack tip might make cracking of an oxide intrusion difficult. It may then survive undamaged over the holding period and grow thicker and denser. Consequently the oxygen diffusion path to the grain boundaries ahead of crack tip is blocked. The cyclic loading then breaks the oxides at the crack tip causing intergranular crack growth and also opens the way for further oxygen diffusion into grain boundaries ahead of the crack tip. Then the intergranular crack advances by repeating this process in a cycle-by-cycle manner, as illustrated in Fig. 8b. Crack tip characterisation under SEM (Fig. 3b) shows that the crack tip opening displacement for the retarded crack is $\sim 500 \mathrm{~nm}$ which is many times larger than the crack tip opening displacement of the fast growing crack. Furthermore, there are no ligaments left behind the crack front from the overall crack morphology observation (Fig. 5b) which is consistent with a large crack opening displacement for this cracking condition. The large crack opening displacement should be associated with the serious deformation around the crack tip which accommodated material displacement. More importantly, such serious deformation is shown by ECCI (Fig. 6b) in this research. Therefore, it is verified that the retardation of 1 hour dwell fatigue crack growth is indeed the result of crack tip blunting.

Moreover, the uncracked thick chromium oxides at the crack tip (Fig. 3b) (see also Ref. [1]) indicate that the crack tip has stayed in such a condition for a long time and no crack extension has happened. All this evidence supports the cycle-by-cycle only crack growth mechanism well.

\section{Summary}

Detailed characterisation of two contrasting types of crack growth behaviour in an advanced nickel-based superalloy has been carried out to investigate a novel, comprehensive proposed mechanism based on the fracture of oxides at the crack tip [1].

The fast intergranular crack growth is indeed brittle. It can form a damage zone because continuous cracking during a dwell period at peak load is very fast and highly selective, strongly depending on local microstructure. The damage zone can be regarded as a crack process zone as a conventional uniform fatigue crack tip does not exist in this situation and d.c.p.c is not able to detect the furthest extent of crack.

The retardation phenomenon, a reduced intergranular crack growth rate, results from a cycle-by-cycle-only advancing procedure because of rapid crack tip blunting in the less creep resistant material.

The main difference between these two mechanisms lays in when and how the oxides intrusion breaks under the influence of the mechanical properties of the matrix and other factors.

The joint support of the UK Engineering and Physical Sciences Research Council (EPSRC) and Rolls-Royce plc under the Strategic Partnership is gratefully acknowledged. We also acknowledge the Henry Moseley X-ray Imaging Facility at The University of Manchester for provision of the X-ray imaging facilities.

\section{References}

[1] Direct communications within a ongoing EPSRCRolls-Royce Strategic Partnership programme. A manuscript has been submitted to Acta Mater

[2] Viskari L, Hörnqvist M, Moore KL, Cao Y, Stiller K. Acta Mater 2013;61:3630

[3] Andrieu E, Molins R, Ghonem H, Pineau A. Mater Sci Eng A 1992;154:21

[4] Goswami T. Int J Fatigue 1999;21:55

[5] Wei RP, Miller C, Huang Z, Simmons GW, Harlow DG. Eng Fract Mech 2009;76:715

[6] Pfaendtner JA, McMahon Jr CJ. Acta Mater 2001;49:3369

[7] Bika D, McMahon Jr CJ. Acta Metall Mater 1995;43:1909

[8] Krupp U, McMahon Jr CJ. J Alloys Compd 2004;378:79

[9] Ma L, Chang K-M. Scr Mater 2003;48:1271

[10] Viskari L, Cao Y, Norell M, Sjberg G, Stiller K. Mater Sci Eng A 2011;528:2570

[11] Kitaguchi HS, Li HY, Evans HE, Ding RG, Jones IP, Baxter G, Bowen P. Acta Mater 2013;61:1968

[12] Miller CF, Simmons GW, Wei RP. Scr Mater 2001; $44: 2405$

[13] Wilkinson AJ, Hirsch PB. Micron 1997;28:279

[14] Krupp U, Kane WM, Liu X, Dueber O, Laird C, McMahon Jr CJ. Mater Sci Eng A 2003;349:213 